\title{
Optical Doppler shift with structured light
}

\author{
Aniceto Belmonte ${ }^{1, *}$ and Juan P. Torres ${ }^{1,2}$ \\ ${ }^{1}$ Technical University of Catalonia, BarcelonaTech, Department of Signal Theory and Communications, \\ 08034 Barcelona, Spain \\ ${ }^{2}$ ICFO-Institut de Ciencies Fotoniques, UPC, Mediterranean Technology Park, \\ 08860 Castelldefels (Barcelona), Spain \\ *Corresponding author: aniceto.belmonte@upc.edu
}

Received July 18, 2011; revised September 14, 2011; accepted October 14, 2011; posted October 17, 2011 (Doc. ID 151305); published November 15, 2011

\begin{abstract}
When a light beam with a transverse spatially varying phase is considered for optical remote sensing, in addition to the usual longitudinal Doppler frequency shift of the returned signal induced by the motion of the scatter along the beam axis, a new transversal Doppler shift appears associated to the motion of the scatterer in the plane perpendicular to the beam axis. We discuss here how this new effect can be used to enhance the current capabilities of optical measurement systems, adding the capacity to detect more complex movements of scatters. (c) 2011 Optical Society of America
\end{abstract}

OCIS codes: $\quad 280.3340,260.0260,140.3300,280.0280$.

Laser remote sensing systems are widely used to monitor moving targets. When a laser beam illuminates an observation region, the interaction of the light beam with the target can modify the phase of the signal reflected back to the receiver, a change that can be used to extract information about the location and velocity of the target.

In a standard laser remote system, with the transmitter and the receiver at the same location, the illuminating optical beam is usually characterized as a Gaussian beam propagating along $\hat{z}$. The corresponding reflected light possesses a time-varying phase $\Psi=2 k z(t)$ that does not depend on the transverse spatial coordinates. Here, $k=2 \pi f / c$ is the wavenumber, $f$ is the frequency of the incident light beam, $c$ is the velocity of light in vacuum, and $2 z(t)$ is the combined propagation distance of the incident and reflected beams. If the target is moving with velocity $\vec{v}$, the reflected signal will show an optical frequency shift $\Delta f_{\|}=k|\vec{v}| \cos \vartheta / \pi$, where $\vartheta$ is the angle between the target's velocity and the line of sight from the object to the observer. This is the usual longitudinal Doppler shift.

The classical nonrelativistic Doppler scheme only allows the direct estimation of the target velocity component along the line of sight, and it does not provide any information about the components of the velocity perpendicular to the direction of propagation of the light beam. The capability to detect transverse velocities is of great interest in fields as diverse as biology microfluidics, microorganism motility [1] , optical coherence tomography medical imaging [2], atmospheric and oceanic turbulence remote sensing [3], and fluid aerodynamics [4].

Here, we present a method for measuring the transverse components of velocity. The key point is to make use of a light beam where each point of the transverse plane is associated with a particular value of the phase of the field (structured light [5]). The phase of the light reflected back to the receiver from a moving target will contain information about the movement of the target at each instant, producing a Doppler shift associated to the change of transverse position. Previous work on measuring transverse velocities has used signal-processing techniques based solely on Doppler measurement along the line of sight for a large set of directions (see, e.g., [6]). If the scatterer is in motion, the frequency of the scattered radiation will in general be different in each direction. Because of the diverse nature of the data sources, postprocessing is required to analyze and correlate the measurements and, in general, the accuracy and spatiotemporal resolution of the measurement might be compromised.

The Doppler shift considered here is of a different origin that the one that can be imparted to a light beam with a Laguerre-Gauss (LG) spatial shape. In this case, the rotation of the beam that causes the Doppler shift can be delivered by using rotating Dove prisms [7]. On the contrary, the observation of the transverse Doppler shift considered here makes use of a stationary structured beam and a fixed detector. Because the target is moving, the light reflected back to the detector changes its phase in accordance to the particular location of the target at each instant. This would be analogous to the Doppler shift captured by a detector that moves in a stationary beam with a phase gradient [8]. The movement of the detector effectively produces a time-varying phase that mimics the phase of the field.

Let us assume that a paraxial light beam illuminates a single, discrete target (e.g., a small particle or scatterer) (see Fig. 1). The incident radiation can be written as

$$
E(\vec{r}, t)=E_{0} \exp \left\{-i\left[2 \pi f t-k z-\Phi\left(\vec{r}_{\perp}\right)\right]\right\}
$$

where $E_{0}$ is the complex amplitude of the beam at the location of the scatterer, $\vec{r}_{\perp}$ describe the transverse position across the beam wavefront, and $\Phi\left(\vec{r}_{\perp}\right)$ is a spatially varying phase that has been imprinted into the beam. These structured light beams can be efficiently generated by making use of appropriately designed spiral or computer-generated holograms [9]. Along these lines, spatial light modulators are becoming an increasingly useful tool, because they allow generation of complex spatial phase and amplitude light patterns and the ability to modify them in a prompt and efficient manner.

When the scatterer passes the observation region with velocity $\vec{v}$, it generates a burst of optical echoes that contributes to the received optical signal. After coherent 

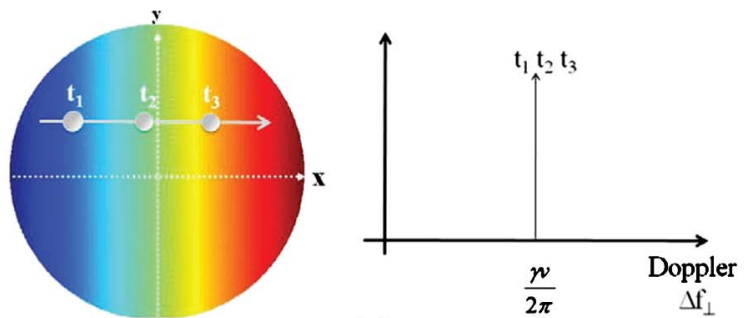

(a)
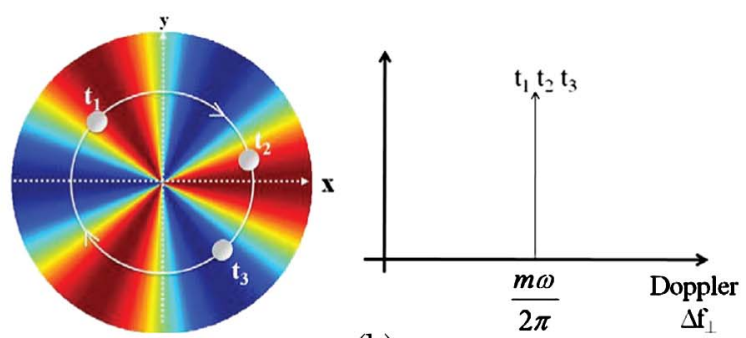

(b)
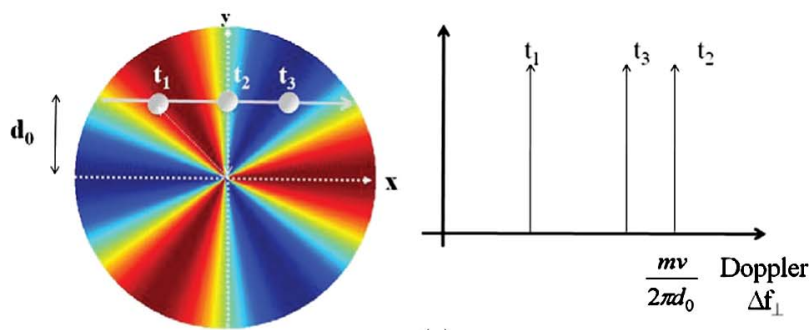

(c)

Fig. 1. (Color online) Sketch of the movement of a target (white solid line) in a beam with a spatially varying phasegradient (as indicated by color scales) and the corresponding Doppler shift of the returned signal for three different times $t_{1}<t_{2}<t_{3}$. (a) Target with constant velocity moves $v$ in a constant phase gradient $\gamma$, induces a time-independent Doppler shift of value $v \gamma /(2 \pi)$. (b) Target rotates with angular velocity $\omega$ in an LG beam and induces a time-independent Doppler shift of value $m \omega /(2 \pi)$. (c) Doppler shift induced by the uniform movement of a scatterer on a twisted LG beam. The frequency shift depends on time $\left(t_{1}, t_{2}, t_{3} \ldots\right)$ as the scatterer moves across the LG beam.

detection and filtering to remove the carrier frequency and its harmonics, we obtain a detected signal $i(t)$ characterizing the optical echo from the target, which can be written as

$$
i(t)=i_{0} \exp \left(i\left\{2 k z(t)+\Phi\left[\vec{r}_{\perp}(t)\right]\right\}\right),
$$

where $\Psi=2 k z(t)+\Phi\left[\vec{r}_{\perp}(t)\right]$ is the time-varying phase of the detected signal and $i_{0}$ is the complex amplitude of the electric signal at the detector, which takes into account the efficiency of the coherent detection process. The coherent detection translates the frequency of the optical backscattered signal to baseband by mixing the input optical signal with an optical local oscillator, and bandpass filtering removes the unwanted noisy signal components [10].

The time rate of the total phase change of the reflected signal is written as

$$
\frac{\partial \Psi}{\partial t}=2 k v_{z}+\nabla_{\perp} \Phi \cdot \vec{v}_{\perp},
$$

where $v_{z}$ is the velocity of the scatterer along the line of sight, $\vec{v}_{\perp}$ is the velocity of the target in the transverse plane, and $\nabla_{\perp} \Phi$ is the phase transversal gradient arising from the spatially varying beam phase. From Eq. (3), we can see that the phase change rate of the reflected signal contains two terms. The first one give rise to the usual longitudinal Doppler shift, i.e., $\Delta f_{\|}=2 v_{z} f / c$. The second term is related to the position of the scatterer in the transverse plane of the illuminating light beam at each time. When the scatterer is moving, its transverse position $\vec{r}_{\perp}$ changes with time and the phase of the detected signal, i.e., $\Phi\left[\vec{r}_{\perp}(t)\right]$ varies accordingly.

This yields a new Doppler frequency shift, due to the transverse velocity of the scatterer, which is written as

$$
\Delta f_{\perp}=\frac{1}{2 \pi} \nabla_{\perp} \Phi \cdot \vec{v}_{\perp} .
$$

It makes use of an optical beam with an appropriately designed phase gradient, and it is proportional to the velocity component of the scatterer along the phase gradient of the transmitted signal. Contrary to the longitudinal Doppler frequency shift, this new transverse Doppler component is not sensitive to the operating optical frequency band.

For illustrative purposes, we analyze the Doppler changes induced by some basic transverse motions of scatterers, i.e., uniform translation and rotation, in some simple optical sensing scenarios. As a first example, let us consider a small scatterer that moves with constant transverse velocity $v$ along an arbitrary direction $\hat{x}$, as shown in Fig. 1(a). To measure this velocity, we can make use of a phase $\Phi=\gamma x$ with a constant gradient $\gamma$. If we make use of Eq. (4), we obtain that the Doppler frequency shift is $\Delta f_{\perp}=\gamma v /(2 \pi)$ [see Fig. 1(a)].

Interestingly, if we consider a light beam with a phase $\Phi=\gamma x^{2} / 2$, whose gradient is now nonuniform, the Doppler shift

$$
\Delta f_{\perp}=\frac{\gamma v}{2 \pi} x
$$

depends on the position of the scatterer. Therefore, the detection of a specific frequency shift gives us information about the specific transverse position of the scatterer, due to the relationship between the Doppler shift and the position caused by the use of a nonuniform phase gradient.

As a second example, let us use LG, one of the simplest light beams showing a phase gradient [11], to detect a rotating scatterer, as shown in Fig. $1(\bar{b})$. LG modes, which appear naturally in laser cavities and have wellknown propagation properties, have been recently the subject of interest for new applications that make use of complex beams [5]. Generally speaking, when an LG beam with azimuthal index $m$ illuminates a single scatterer, the phase $\Psi=2 k z+m \varphi$ of the backscattered signal depends on the cylindrical azimuthal angle $\varphi$. For a moving scatterer, the phase of the twisted beam 
produces a transverse phase gradient written as $\nabla_{\perp} \Phi=$ $m \hat{\varphi} / \rho$. If we make use of Eq. (4), we obtain that the time rate of the echo phase signal due to the transverse velocity of the scatterer produces a frequency shift

$$
\Delta f_{\perp}=\frac{m \omega}{2 \pi},
$$

where $\omega$ is the instantaneous angular velocity of the scatterer.

When the discrete target has a circular uniform motion around the beam axis, the spectral content of the returned signal from the scatterer is characterized by a single spectral line with separation $m \omega /(2 \pi)$ from the center frequency [see Fig. 1(b)]. If the illuminating beam is rotated at angular frequency $\Omega$, it is not difficult to see that the Doppler frequency shift is now $m(\omega+\Omega) /(2 \pi)$. It is thus apparent that using a twisted beam rotated at angular frequency $\Omega$ is equivalent to "angular" heterodyning the coherent detected signal a frequency $m \Omega /(2 \pi)$.

If the single target would move on an LG beam with an uniform movement (as shown in Fig. 1(c), lower row), a variable in time Doppler shift would be observed. The velocity of the scatterer can be expressed in cylindrical coordinates as $\vec{v}=v(\hat{\rho} \cos \varphi-\hat{\varphi} \sin \varphi)$, where we have assumed, without loss of generality, that the scatterer moves along a path parallel to the direction $\hat{x}$ at a distance $d_{0}$ from the axis $y=0$. By making use of the relationship $\sin (\varphi)=d_{0} / \rho$, we obtain an instantaneous Doppler frequency shift

$$
\Delta f_{\perp}=\frac{m d_{0} v}{2 \pi \rho^{2}} .
$$

As the scatterer approaches, passes, and recedes from the closest point to the center of the beam, the frequency shift of the returned signal increases, reaches its maximum, and then decreases [see Fig. 1(c)]. The maximum value $m v /\left(2 \pi d_{0}\right)$ is reached when $\rho=d_{0}$, i.e., when $\varphi=\pi / 2$.

In conclusion, we have shown that the use of structured light beams with appropriately designed phase gradients allows to retrieve, apart from the usual velocity of a target along the line of sight, transverse velocity components from the target motion.

In particular, applied to a monostatic optical radar, it might allow studying atmospheric wake turbulence [4]. For example, during takeoff and landing, a typical large passenger airplane creates two trailing vortices of a few meters diameter and some tens of meters per second azimuthal velocity. Using an LG beam with winding number $m=10$ would produce Doppler shifts of several tens of hertz.

In a different application, the approach proposed here can be considered for the measurement of the motility of single-celled and simple multicellular organisms. For example, for a typical value of motility [1] of a biological specimen of tens of micrometers per second, a linear spatial modulation of $\gamma \sim 1 \mu \mathrm{m}^{-1}$ would yield a Doppler frequency shift of some tens of hertz. This scheme can also be used to measure fluid flow in live tissue, where the possibility of inducing tiny phase gradients that would not affect the in vivo system under study can be of great interest. For instance, the diagnosis of certain important eye diseases can be assessed by observing abnormal retinal blood flow [2]. Typical blood flow velocities in the retina are in the range of some tens of $\mathrm{mm} / \mathrm{s}$. Using phase gradients of some $\gamma \sim 0.1 \mu \mathrm{m}^{-1}$, would result in Doppler shifts of several kilohertz.

The results developed require no statistical model for the velocity components and allow estimating scatterer transversal velocity components from Doppler shift measurements. We should notice that in certain important situations, Doppler optical echoes may be composites of signals from a dense array of single scatterers, each of which moving with identical velocity. The Doppler spectrum will then be the result of a power-weighted distribution of echoes frequencies from the scatterers illuminated by the optical beam. In that case, we would need to consider the specific spatial intensity distribution of the beams and the specific distribution of scatterers.

This work was supported by the government of Spain (grants TEC2009-10025 and FIS2010-14831). The project PHORBITECH acknowledges the financial support of the Future and Emerging Technologies (FET) Programme, under FET-open grant number: 255914. This work was also supported in part by the Fondo de Cooperación Internacional en Ciencia y Tecnología (FONCICYT) project 94142 and Fundacio Privada Cellex Barcelona.

\section{References}

1. Y. R. Chemla, H. L. Grossman, T. S. Lee, J. Clarke, M. Adamkiewicz, and B. B. Buchanan, Biophys. J. 76, 3323 (1999).

2. Y. Wang, B. Bower, J. Izatt, O. Tan, and D. Huang, J. Biomed. Opt. 12, 041215 (2007).

3. W. B. Grant, in Tunable Laser Applications, F. J. Duarte, ed. (CRC, 2009).

4. M. Harris, R. Young, F. Köpp, A. Dolfi, and J. Cariou, Aerospace Sci. Technol. 6, 325 (2002).

5. J. P. Torres and L. Torner, eds., Twisted Light (Wiley, 2010).

6. F. Durst, B. M. Howe, and G. Richter, Appl. Opt. 21, 2596 (1982).

7. J. Courtial, K. Dholakia, D. A. Robertson, L. Allen, and M. J. Padgett, Phys. Rev. Lett. 80, 3217 (1998).

8. M. Vasnetsov, J. P. Torres, D. Petrov, and L. Torner, Opt. Lett. 28, 2285 (2003).

9. N. R. Heckenberg, R. McDuff, C. P. Smith, and A. G. White, Opt. Lett. 17, 221 (1992).

10. J. W. Goodman, Statistical Optics (Wiley-Interscience, 2000).

11. R. Loudon, Phys. Rev. A 68, 013806 (2003). 